\title{
Iron-based Metal Organic Frameworks: Size Controlled Synthesis and Characterization
}

Karla Guadalupe Espinoza-Montoya, Andrea Guadalupe López-Vega, Paul Zavala Rivera, Armando Lucero-Acuña, Ofelia del Carmen Hernández Negrete, Daniel Fernández-Quiroz and Cindy GutierrezValenzuela

Universidad de Sonora, Hermosillo, Sonora, Mexico

Metal organic frameworks (MOF) are a unique type of hybrid materials composed of metal-oxide units joined by an organic linker, they can be formed by a diversity of component resulting in crystalline structures with unique structural features ${ }^{[1,2]}$. MOF have become of great interest as they have shown great potential for drug delivery, catalysis, purification processes, chemical separation, storage and capture of molecules, sensors, pollution control, and recently, in the manufacture of batteries, super-capacitors and solar cells ${ }^{[3-7]}$. Iron-based MOF nanocrystals are known for their non-toxic nature, high drug adsorption capacity and by being highly flexible frameworks. The resulting MOF characteristics can be affected by a variety of parameters, as the rate of nucleation is affected and therefore the size and aspect ratio (length/width) of nanocrystals. The use of acetic acid during synthesis of MOF can affect the size of the final nanocrystals ${ }^{[1,4,6,8,9]}$.

The Fe-MIL-88B-NH $2 \mathrm{MOF}$ can be synthesized using ferric chloride hexahydrate $\left[\mathrm{FeCl}_{3} \mathrm{Z}_{2} 6 \mathrm{H}_{2} \mathrm{O}\right]$ as the metal source, 2-aminoterephthalic acid $\left(\mathrm{H}_{2} \mathrm{~N}-\mathrm{BDC}\right)$ as the organic linker and pluronic $\mathrm{F} 108$ as stabilizer $^{[3,10,11]}$. Acetic acid can be used optionally with the purpose of deprotonating the carboxylic linkers during the synthesis and controlling the size of the final $\mathrm{MOF}^{[3]}$. In this work, the following compound molar ratio was used: $\mathrm{Fe}^{3+}: \mathrm{H}_{2} \mathrm{~N}-\mathrm{BDC}: \mathrm{H}_{2} \mathrm{O}: \mathrm{F} 108: \mathrm{CH}_{3} \mathrm{COOH}=1: 0.5: 1255: 0.02: \mathrm{Y}$, where $\mathrm{Y}$ was varied from 0 to 8 to 16 for MOF A, B and C, respectively. After hydrothermal synthesis, the solid product is recovered and washed several times with ethanol by vacuum filtration to remove the surfactant and excess reagents. Finally, product dried using an oven and overnight.

Characterization of MOF include SEM and TEM for visualization of size and morphology, FTIR and XRD for composition and structure confirmation. Also, the BET technique can be used to measure surface area and pore size. Figure 1 and 2 present SEM and TEM images obtained for MOF A, B and C for each technique. In these micrographs, bipyramid hexagonal prism crystals can be appreciated with sizes (long/width) of 119/71nm $\pm 13 / 16$ for MOF A; 273/111nm $\pm 21 / 4.5$ for MOF B; and 775/213nm $\pm 84 / 44$ for MOF C. The increase in size is noted as acetic acid ratio is increased. FTIR spectrum for the MOF A, $\mathrm{B}$ and $\mathrm{C}$ are presented in Figure 3. It is noted that the amine group is shown as well as the Fe-O bond, confirming the formation of the Fe-MIL-88B-NH 2 . Figure 4 presents the XRD results where the diffraction lines in $2 \theta=10.1^{\circ}(002), 16.8^{\circ}(200), 17.6(202)$ represent the iron chloride phase by JCPDS card No. [77-998], and the next phase represented is the terephthalic acid in $2 \theta=12.1^{\circ}(102), 17.4^{\circ}(103)$ by JCPDS card No. [31-1916]. Surface area obtained for MOF A, B and C decreased from 16.2 to 8.8 and $6.9 \mathrm{~m}^{2} / \mathrm{g}$ for each of the samples, respectively. In the same way, the pore volume decreased, ranging from 0.08867 and $0.01985 \mathrm{~cm}^{3} / \mathrm{g}$ for the three preparations. Pore size decreased as well, from 10.9 to 8.1 to 5.8 $\mathrm{nm}$ respectively. Adsorption/desorption isotherms were presented as a type IV isotherm corresponding to mesopores with pore sizes of 2-50 nm, which also correspond to the results obtained. 
There are several parameters that can affect or influence the rate of nucleation of the MOF during synthesis. These variations can result in a variety of size and aspect ratio of the resulting structures. The addition of acetic acid to the preparation of Fe-MIL-88B-NH $2 \mathrm{MOF}$ can be used to control the size of the MOF: the higher the ratio of iron:acetic acid, the greater the size of the crystal.

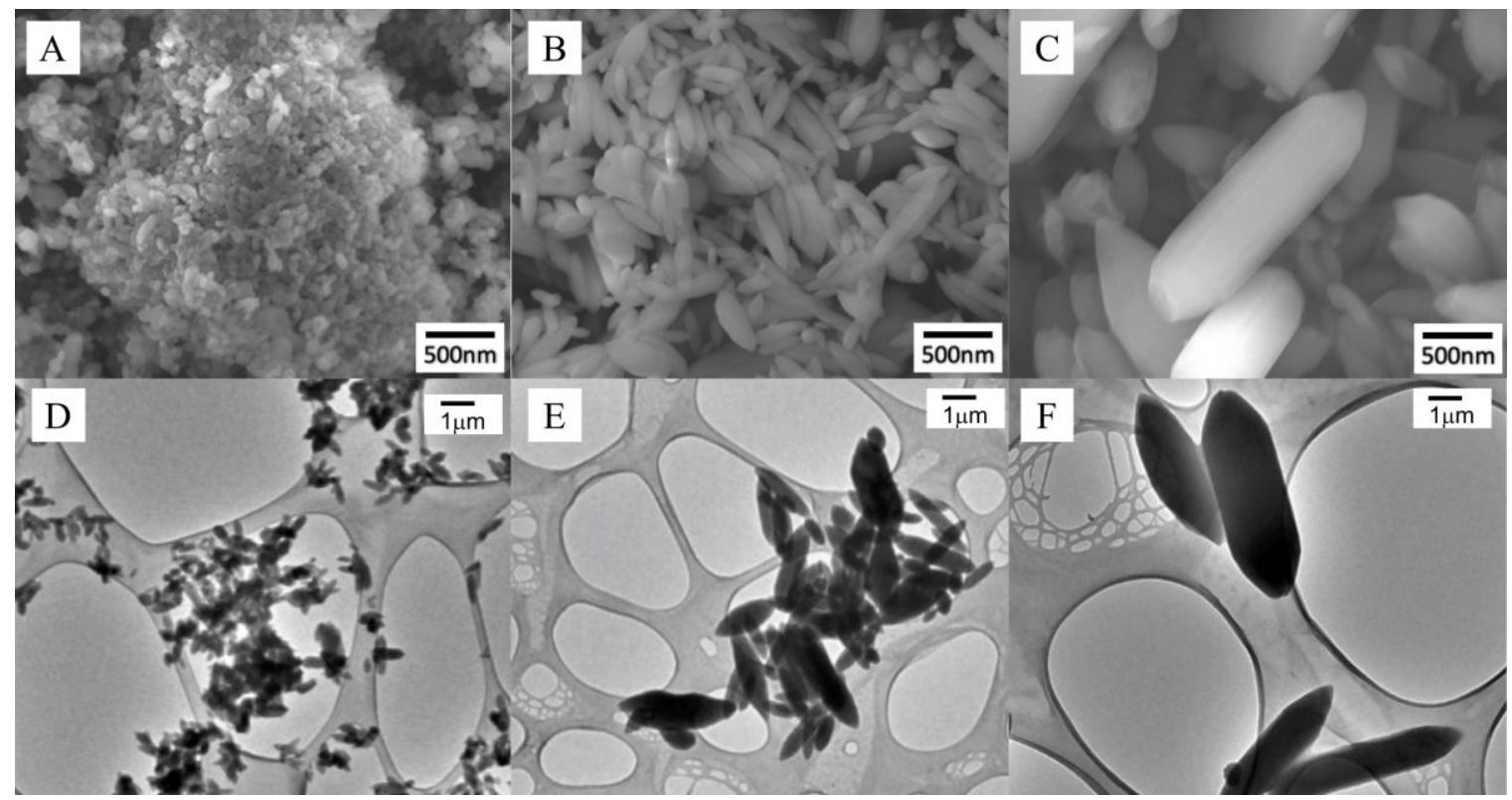

Figure 1. SEM micrograph from (A) MOF A, (B) MOF B and (C) MOF C Fe-MIL-88B-NH2. TEM micrograph from Fe-MIL-88B-NH2 (D) MOF A, (E) MOF B and (F) MOF C.
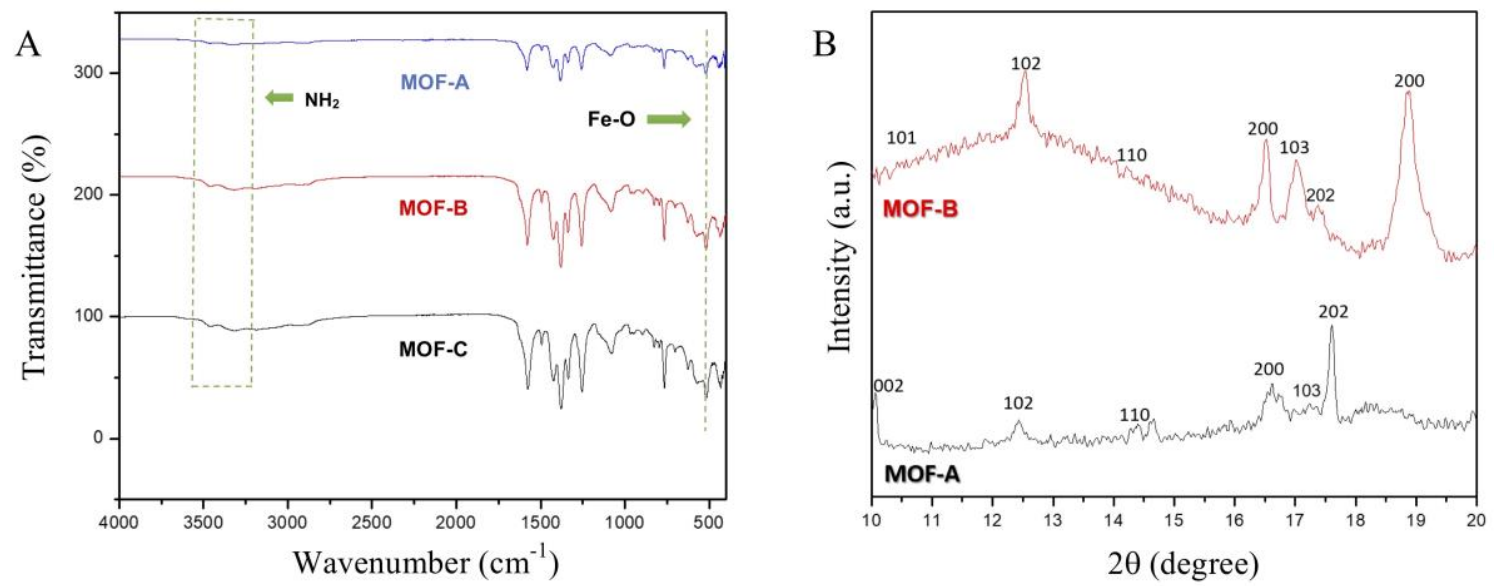

Figure 2. FTIR spectrum (A) obtained for Fe-MIL-88B-NH2 MOF A, B and C and XRD spectrum obtained for Fe-MIL-88B-NH2 MOF A and B.

\section{References}

[1] J. Tang, J. Wang, Chem. Eng. J. 2018, 351, 1085.

[2] G. Liu et al., Carbohydr. Polym. 2017, 175, 584.

[3] M. Pham, G. Vuong, A. Vu, T. Do, Langmuir 2011, 27, 15261.

[4] S. Sorribas, C. Téllez, Grup. Español Carbón 2016, 19.

[5] D. Di et al., J. Phys. Chem. Lett. 2015, 6, 446.

[6] N. Liédana, P. Lozano, A. Galve, C. Téllez, J. Coronas, J. Mater. Chem. B 2014, 2, 1144. 
[7] R. K. Ameta et al., J. Mol. Liq. 2018, 268, 677.

[8] J. He, Y. Zhang, X. Zhang, Y. Huang, Sci. Rep. 2018, 8, 1.

[9] B. Ghalei et al., Nat. Energy 2017, 2.

[10] L. A. Lu, Y. S. Ma, M. Kumar, J. G. Lin, Chem. Eng. J. 2011, 166, 150.

[11] B. Bazan et al., Macla. Rev. la Soc. Española Mineral. 2012, 2, 162. 